\title{
UK experience of liver transplantation for erythropoietic protoporphyria
}

\author{
Joanna K. Dowman • Briget K. Gunson • \\ Darius F. Mirza $\cdot$ Mike N. Badminton • \\ Philip N. Newsome • \\ on behalf of the UK Liver Selection and Allocation \\ Working Party
}

Received: 2 December 2010 /Revised: 2 December 2010/Accepted: 23 December 2010 /Published online: 8 February 2011

(C) SSIEM and Springer 2011

\begin{abstract}
Erythropoietic protoporphyria (EPP) is characterised by excess production of free protoporphyrin from the bone marrow, most commonly due to deficiency of the enzyme ferrochelatase. Excess protoporphyrin gives rise to the cutaneous photosensitivity characteristic of the disease, and in a minority of patients leads to end-stage liver disease necessitating liver transplantation (LT). There is limited information regarding the timing, impact and long-term outcome of LT in such patients, thus we aimed to identify the indications and outcomes of all transplants performed for EPP in the UK using data from the UK Transplant Registry. Between 1987 and 2009, five patients underwent LT for EPP liver disease. Median follow-up was 60 months, and there were two deaths at 44 and 95 months from causes unrelated to liver disease. The remaining recipients are alive
\end{abstract}

Communicated by: Georg Hoffmann

Competing interest: None declared.

J. K. Dowman $(\varangle) \cdot$ B. K. Gunson • P. N. Newsome $(\square)$

Centre for Liver Research, 5th floor,

Institute of Biomedical Research, University of Birmingham,

Edgbaston,

Birmingham B15 2TT, UK

e-mail: j.k.dowman@bham.ac.uk

P. N. Newsome

e-mail: p.n.newsome@bham.ac.uk

J. K. Dowman · B. K. Gunson • D. F. Mirza • P. N. Newsome

The Liver Unit, Queen Elizabeth Hospital Birmingham,

Edgbaston,

Birmingham B15 2TH, UK

M. N. Badminton

Department of Infection, Immunity and Biochemistry,

School of Medicine, Cardiff University,

Cardiff CF14 4XN, UK at 22.4 years, 61 months and 55 months after transplant. A high rate of postoperative biliary stricturing requiring multiple biliary interventions was observed. Recurrent EPP-liver disease occurred in $4 / 5(80 \%)$ of patients but graft failure has not been observed. Given the role of biliary obstruction in inducing EPP-mediated liver damage, we suggest that consideration should be given for construction of a Roux loop at the time of transplant. Thus we demonstrate that although EPP liver transplant recipients have a good long-term survival, comparable to patients undergoing LT for other indications, biliary complications and disease recurrence are almost universal, and bone marrow transplantation should be considered where possible.

$\begin{array}{ll}\text { Abbreviations } \\ \text { ALAS2 } & \text { Aminolaevulinic acid synthase } 2 \\ \text { BMT } & \text { Bone marrow transplantation } \\ \text { EPP } & \text { Erythropoietic protoporphyria } \\ \text { ERCP } & \text { Endoscopic retrograde cholangiopancreatography } \\ \text { ESLD } & \text { End stage liver disease } \\ \text { INR } & \text { International normalised ratio } \\ \text { LT } & \text { Liver transplantation } \\ \text { MARS } & \text { Molecular adsorbent recycling system } \\ \text { MELD } & \text { Model for end stage liver disease } \\ \text { NHS } & \text { National Health Service } \\ \text { UNOS } & \text { United Network for Organ Sharing } \\ \text { XLDPP } & \text { X-linked dominant protoporphyria }\end{array}$

\section{Introduction}

The erythropoietic porphyrias include two disorders characterised by excess production of free protoporphyrin from the bone marrow, due to either deficiency of the enzyme ferrochelatase (erythropoietic protoporphyria; EPP MIM\#177000) (Elder et al. 2009; Todd 1994), or a gain of 
function mutation in the enzyme erythroid ALA synthase (ALAS2) (X-linked dominant protoporphyria; XLDPP MIM\#300752). The latter is less common (Elder et al. 2009) but appears to carry a higher risk of liver disease (Whatley et al. 2008). Photo-excitation of protoporphyrin in the skin leads to the painful photosensitivity characteristic of the disease (Todd 1994). Increased biliary protoporphyrin excretion exerts toxic effects on hepatobiliary structure and function (Avner et al. 1981;Morton et al. 1988), although advanced, progressive liver disease manifests in only a small minority (Anstey and Hift 2007;Doss and Frank, 1989; Meerman 2000). As yet there are no modifiable risk factors for the development of liver disease in protoporphyria which would allow those at highest risk to be closely monitored. However, the risk appears to be increased in the $4 \%$ of patients who are homozygotes or compound heterozygotes for rare ferrochelatase (FECH) mutations (Elder et al. 2009; Whatley et al. 2004) and in XLDPP (Whatley et al. 2008). A cross-sectional study of protoporphyria in the UK has calculated the minimum prevalence of EPP in the UK to be 1:143,000 (Holme et al. 2006). Of the 223 UK patients recruited to the study, $4(1.8 \%)$ had severe liver disease that had required management in a liver unit (Holme et al. 2006). Although medical management is the mainstay of treatment for the great majority of patients, liver transplantation should be considered for patients with end-stage liver disease.

Mechanisms of protoporphyrin hepatotoxicity in EPP in experimental models include formation of cytotoxic bile which damages biliary epithelium leading to biliary fibrosis (Meerman et al. 1999), impaired bile formation (Avner et al. 1981) and alterations in hepatic membrane enzyme activity (Avner et al. 1983).

Our group has previously reported the short term follow up of two patients undergoing liver transplantation for EPP in a single centre (Herbert et al. 1991; Seth et al. 2007). No other adult liver transplants have been reported for this indication in the UK. Using data from the UK Transplant Registry maintained by NHS Blood and Transplant on behalf of the transplant community, we here describe a larger national series with longer term follow up which includes all liver transplants performed for EPP in the UK from 1987-2009.

\section{Methods}

This is a retrospective study of all known patients in the UK transplanted for EPP liver disease. They were identified by searching the UK Transplant Registry. The diagnosis of EPP was made by clinical features, elevated erythrocyte and plasma free protoporphyrin levels and histology of explanted liver.

Data collected included patient demographics, preoperative laboratory results and clinical status, date of transplant, long term graft and patient survival, cause of death where relevant, and immunosuppressive protocol. Postoperative complications were also recorded. Missing data were obtained where possible by review of case notes or communication with the respective liver unit. Patient survival was defined as the time between initial transplant and patient death.

The MELD (model for end-stage liver disease) score was calculated for each patient in this study to assess the severity of liver disease. The MELD is a validated marker of risk of early death without transplantation (Neuberger et al. 2008), and is used by the United Network for Organ Sharing (UNOS) to determine priority for adult organ allocation. The score is calculated using the variables of serum bilirubin, international normalised ratio (INR) and creatinine, and the survival benefit for transplantation is for patients with a MELD score $>16$ (Neuberger et al. 2008).

\section{Results}

Between 1987 and 2009, five patients were identified to have undergone liver transplantation for protoporphyria in the UK. Erythrocyte porphyrin biochemistry results were available for four patients and consistent with the diagnosis of EPP (increased free protoporphyrin, normal zinc protoporphyrin fraction). Transplants were performed across three different liver transplant centres.

Three $(60 \%)$ patients were male, and median age at transplant for EPP was 40 years (range 17-59 years). Two transplants took place in 1987 and 1992, and the remaining three between 2003 and 2005 (Table 1).

Biliary reconstruction at the time of transplant was performed using a duct-to-duct anastomosis in the four $(80 \%)$ patients for whom these data were available. All patients were commenced on combined calcineurininhibitor based immunosuppressive therapy, with the individual protocols reflecting the more recent trend towards use of tacrolimus in place of cyclosporine in such regimens. Two (40\%) of EPP transplant recipients required readmission during the first year due to acute rejection, one of whom also developed evidence of chronic rejection.

Indication for transplant

In EPP, the indication for liver transplantation was the development of protoporphyrin-induced end-stage liver disease, defined by liver histology and markedly abnormal liver function. Median MELD score at the time of listing was 22 (range 19-25), which is comparable to adults undergoing liver transplantation for other indications (Freeman 2008). In our centre, one patient also suffered severe photosensitivity, gallstones, and recurrent acute 
crises of abdominal pain and hepatitis requiring regular treatment with both hemin and opiates. Recurrent red cell transfusions in this patient, performed to maintain haemoglobin and thus reduce the drive for haem synthesis and consequent protoporphyrin production, necessitated additional desferrioxamine therapy in order to prevent iron overload. With no evidence of overt blood loss, the chronic low-grade anaemia observed here may have been related to haemolysis, iron deficiency and/or chronic disease. A second patient at our centre presented with photosensitive dermatitis, and underwent transplantation four years after diagnosis for end-stage EPP-liver disease after a rapid deterioration with encephalopathy and jaundice.

Explant histology

Explant histology was available for three patients. One report described a macroscopically hard and dark liver typical of EPP, with histological analysis revealing early micronodular cirrhosis, large amounts of brown pigment in Kupffer cells, and dilated biliary canaliculi, hepatocytes and bile ducts. A second liver explant was macroscopically dark brown and nodular, with microscopic analysis revealing early cirrhosis with a biliary pattern, and grade 3 parenchymal siderosis. Protoporphyrin crystals were present with pigment in hepatocytes, Kupffer cells and bile canaliculi. The third liver explant was reported to be macroscopically enlarged and greenish-black in colour. Histology revealed cirrhosis with marked cholestasis, grade 1 siderosis, and the presence of brown pigment deposits in bile ducts, Kupffer cells and hepatocytes with red birefringence consistent with EPP.

\section{Survival}

Median follow-up was 60 months. Two deaths occurred at 44 and 95 months, and three patients remain alive at 22.4 years, 61 months and 55 months after transplant. One patient died from cerebral tuberculosis; the other cause of death was unspecified but not related to the liver.

\section{Disease recurrence}

In EPP liver transplantation does not alter the excessive production of protoporphyrin in erythroid cells from the bone marrow. Recurrence of liver damage is therefore common, and histologically proven recurrent EPP liver disease occurred within 2 years of transplant in four (80\%) of these patients. Two had recurrence by 4 months, and one as early as 24 days post-transplant. Recurrence was usually associated with abnormal liver function and complications such as biliary sludge/stones, however graft failure from recurrent disease has not yet been observed in this cohort. Biopsy in recurrent disease typically showed reaccumulation 
of protoporphyrin crystals, pigmentation, and cholestasis, with or without fibrosis. Absence of any features of recurrent EPP liver disease in a single patient was confirmed by biopsy 16 years post-transplant. This individual had been taking the bile acid sequestrant cholestyramine throughout the period of follow-up.

As the only potentially curative treatment for this disorder, bone marrow transplantation (BMT) was performed in one patient with recurrent EPP liver disease, in whom neither dialysis nor molecular adsorbent recycling system (MARS) had demonstrated a beneficial effect on erythrocyte protoporphyrin levels. Although BMT did lead to cure of the EPP, as demonstrated by a dramatic fall in erythrocyte protoporphyrin levels and a fully functioning liver, this patient unfortunately died 2.7 years later from cerebral tuberculosis. BMT has been considered but not undertaken in two other patients in this cohort, one of whom died from unrelated causes.

\section{Complications}

Biliary complications, primarily stones, sludge and strictures, are frequently observed in EPP. Four (80\%) of the patients in this cohort experienced such complications after liver transplantation, which were usually evidenced biochemically by worsening liver function of a cholestatic nature. All of these patients have required multiple interventions for biliary strictures and/or stones/sludge (Table 1).

Second-degree skin burns due to peri-operative light exposure were observed in one patient, who also experienced a post-operative biliary leak and later required ERCP and sphincterotomy for biliary sludge/stone related cholestasis. This patient eventually underwent a biliary Roux-en$\mathrm{Y}$ reconstruction 14.5 years post-transplant following an episode of cholangitis related to a common bile duct stone and anastomotic stricture. Liver biopsy repeated several months after this intervention confirmed disappearance of the previously observed features of chronic biliary obstruction, and no further biliary complications have occurred in the subsequent 8 years of follow-up. Another patient with particularly severe recurrent EPP-mediated liver damage developed a post-transplant biliary anastomotic stricture with consequent deterioration in cutaneous symptoms, liver function tests and erythrocyte protoporphyrin levels. ERCP with dilatation and stenting has been performed on several occasions with notable improvement, and consideration given to the possibility of definitive biliary reconstructive surgery. Four years after transplantation an episode of late acute rejection in this patient, in the setting of worsening EPP-induced liver damage with biliary complications, led to a rise in bilirubin to over $450 \mu \mathrm{mol} / \mathrm{L}$. Liver biopsy showed evidence of chronic hepatitis with moderate fibrosis, biliary features and protoporphyrin crystals con- sistent with significant EPP-induced liver damage. Manual red cell exchange was successfully undertaken in this individual (Fig. 1), with marked biochemical and symptomatic improvement. In addition to red cell exchange, haemoglobin levels were maintained $>12 \mathrm{~g} / \mathrm{dl}$ (Fig. 1).

Of the three remaining patients, two individuals with biopsy-proven recurrent EPP liver disease required recurrent admissions for ERCP to treat biliary complications (stricture/sludge/stones). One of these patients was being considered for BMT due to iron overload at the time of her death, which occurred at 44 months post-transplant from a cause unrelated to the liver.

The remaining patient had biopsy-proven EPP-liver disease recurrence by day 24 post-transplant. Although no biliary complications were observed, this patient underwent bone marrow transplantation (BMT) one year after LT, following which a significant fall in plasma porphyrin levels was observed together with a fully functioning liver, suggesting this was indeed curative. However complications of immunosuppression and sepsis eventually culminated in death 2.7 yrs after BMT.

\section{Discussion}

In this study we report a long-term series of patients undergoing liver transplantation for EPP in the UK, which includes all adult patients transplanted for this indication between 1987 and 2009.

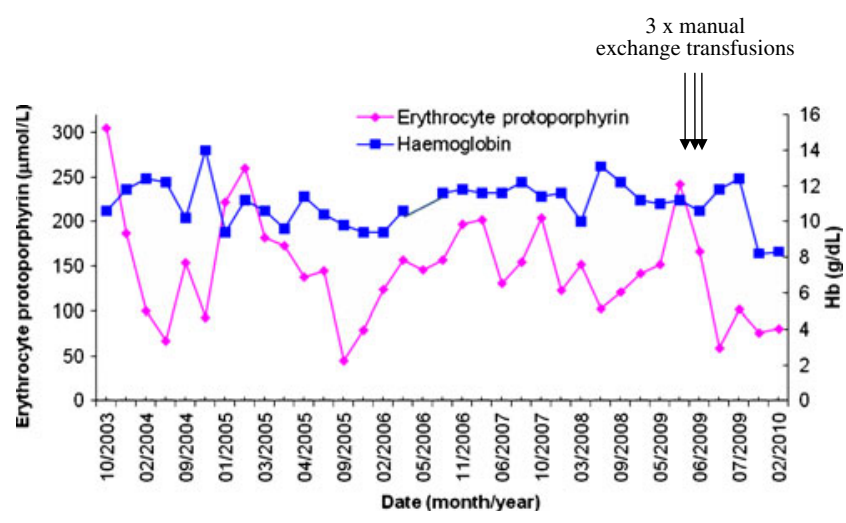

Fig. 1 Relationship between haemoglobin and disease activity and effect of manual red cell exchange on erythrocyte protoporphyrin levels in a patient with post-transplant EPP recurrence. Erythrocyte protoporphyrin levels decreased rapidly following LT in 2005, before rising again in association with disease recurrence. Reductions in haemoglobin correspond with increasing erythrocyte protoporphyrin levels as a result of the increased drive for haem synthesis, highlighting the importance of maintaining an adequate haemoglobin level $>\sim 12 \mathrm{~g} / \mathrm{dl}$. Repeated manual red cell exchange transfusions in 2009 resulted in a rise in haemoglobin and reduction in erythrocyte protoporphyrin levels associated with symptomatic improvement. The standard protocol involved removal of $250 \mathrm{ml}$ of blood via a central vein and replacement with $250 \mathrm{ml}$ normal saline and 1 unit of packed red cells 
In contrast to acute porphyrias, where the majority of severely affected patients are female, in this study there was no significant gender difference in patients undergoing transplantation for EPP. These findings are consistent with the US series of 20 patients where a 3:2 male:female ratio was observed(McGuire et al. 2005). It remains possible that this slight male predominance may reflect the increased frequency of XLDPP in males, which may also be more likely to cause hepatopathy, (Elder et al. 2009) although ferrochetolase gene abnormalities were present in each of the ten patients in this study in whom DNA analysis was performed. (McGuire et al. 2005).

Median age at transplant was 40 years which is considerably younger than the traditional elective liver transplant population (van der Meulen et al. 2007). Three EPP recipients $(60 \%)$ remain alive at between 55 months and 22.4 years and there were two deaths at 44 and 95 months from causes not directly related to the porphyria. This data is again in keeping with reports of patients transplanted for this indication in the US, where a $69 \%$ patient and graft survival was seen (McGuire et al. 2005). It has been reported that liver disease is more prevalent in patients with autosomal recessive EPP (Elder et al. 2009; Whatley et al. 2008) and XLDPP (Whatley et al. 2008). In this cohort, all patients had an erythrocyte protoporphyrin screen to identify the presence of free and or zinc protoporphyrin, and XLDPP was considered unlikely because of the absence of the typical pattern of increased zinc and free protoporphyrin associated with this disorder. Although the proportion of transplanted patients with XLDPP is not presently known, and this subgroup of patients does warrant more careful monitoring, it is likely that these patients account for a minority of the liver disease burden in EPP.

As EPP patients undergo liver transplantation for endstage liver disease this is reflected by MELD scores which are comparable to patients transplanted for other diagnoses (Freeman 2008). Although LT is associated with good survival rates, it does not correct the underlying enzyme defects and excessive protoporphyrin production in the bone marrow continues (Samuel et al. 1988) leading to almost universal recurrence of liver damage. In our series $80 \%$ of patients had histological evidence of recurrent liver damage, of which one case is particularly advanced. Disease recurrence was usually also reflected in a deterioration in liver function tests, with a rise in bilirubin and other markers of cholestasis. This is similar to the US experience where $65 \%$ had recurrence and $15 \%$ required re-transplantation (McGuire et al. 2005). Attention should therefore be focussed on strategies to minimise the extent of recurrent liver damage after transplantation.

Biliary complications are very common after liver transplantation for EPP, and were observed in $80 \%$ of patients in this cohort. A $45 \%$ biliary complication rate was reported in the US series (McGuire et al. 2005) which, although lower than our experience, remains significantly higher than the reported rate of $15-30 \%$ observed in the general liver transplant population.(Welling et al. 2008) Biliary outflow obstruction, whether from anastomotic strictures or disease-related accumulation of stones/sludge, is particularly detrimental in these patients as impairment of bile flow causes hepatic protoporphyrin accumulation and promotes recurrence of EPP in the graft (McGuire et al. 2005). The early identification and correction of such complications is therefore essential. One patient transplanted at our centre underwent Roux-en-Y biliary reconstruction several years after transplantation following recurrent biliary obstructive complications. This procedure led to complete histological resolution of biliary features, with no further biliary complications during eight subsequent years of follow-up. Although there is little direct experience in the setting of EPP, in the general liver transplant population Roux-en-Y choledochojejunostomy at the time of transplant has been associated with a significantly lower rate of biliary complications compared with conventional common duct anastomosis.(Leonardi et al. 2005; Welling et al. 2008). Thus we would suggest that biliary reconstruction with a Roux loop at the time of liver transplant may further reduce the rate of biliary complications and hence decrease EPP-mediated liver damage in the allograft.

The occurrence of light-induced skin burns in the first patient transplanted for EPP in our centre led to the use of intra-operative light filters during subsequent procedures to prevent light-induced skin and intestinal damage (McGuire et al. 2005; Seth et al. 2007), although minor burns may still occur (McGuire et al. 2005). However this could be reduced by adopting a recently proposed combination of light filters (Wahlin et al. 2008). In our experience such filters do not cause significant distortion of vision or interference with surgery(Seth et al. 2007).

The presence of anaemia, from haemolysis, iron deficiency or chronic disease, drives the haem synthesis pathway, further increasing the production of hepatotoxic protoporphyrins. It is therefore recommended that adequate haemoglobin levels $(\geq 12 \mathrm{~g} / \mathrm{dl})$ are maintained to reduce protoporphyrin production. Recurrent red cell transfusion has been demonstrated to reduce erythrocyte and plasma protoporphyrin levels in association with improvements in photosensitivity and hepatic function, although does carry the risk of iron overload (Dobozy et al. 1983; Spiva and Lewis 1984;van Wijk et al. 1988; Wahlin et al. 2007). Other therapeutic options which may be considered include inhibiting protoporphyrin production with intravenous hemin infusion, (Do et al. 2002), and removal of protoporphyrin using plasmapheresis (Do et al. 2002), and/or red 
cell exchange (Eichbaum et al. 2005). Both hemin infusion and plasmapheresis have been demonstrated in a small number of patients to improve plasma and erythrocyte protophorphyin levels in addition to liver biochemistry and histology(Dellon et al. 2002;Do et al. 2002;Dobozy et al. 1983; Reichheld et al. 1999). Similar improvements have been demonstrated with red cell exchange transfusion (Eichbaum et al. 2005) although such treatment is seldom used and generally reserved for severe or rapidly deteriorating cases (Anstey and Hift 2007). Whilst red cell exchange has been helpful in one of our cases with severe recurrent EPP-mediated liver damage, plasmapheresis was not found to be of benefit in another of the EPP patients in this cohort.

Bone marrow transplantation (BMT) is at present the only treatment able to correct the underlying bone marrow defect resulting in excessive protoporphyrin production (Fontanellas et al. 2000; Wahlin et al. 2007). BMT has been successfully used early post-transplant to stabilise or prevent recurrent EPP liver disease (Rand et al. 2006) and, although experience is limited, this option may be considered in patients undergoing LT for EPP if a compatible related donor is available. This is reinforced by survival data for BMT for non-malignant disease, which range from a 3-year survival rate of $70-90 \%$ in matched sibling donors to $36-65 \%$ in unrelated donors (Appelbaum 2003), underlining the importance of identifying sibling donors. The single BMT undertaken in our cohort was performed one year after liver transplantation due to rapid EPP disease recurrence. Although BMT did cure the EPP, this patient unfortunately died 32 months later, with a fully functioning liver, from cerebral tuberculosis related to complications of immunosuppression. Concurrent liver and bone marrow transplantation has not to our knowledge been attempted.

In conclusion, patients transplanted for EPP have a good long-term survival, although given the high rate of biliary complications and recurrent EPP-mediated liver damage we would suggest consideration of Roux loop formation at the time of transplant. BMT should be considered for patients with sibling donors as this will result in cure of the EPP.

Acknowledgements The authors would like to acknowledge the help from staff at Royal Free Hospital, London and Addenbrooke's Hospital, Cambridge for the provision of additional information.

Funding source JK Dowman is a Wellcome Trust Clinical Research Fellow and is therefore receiving funding from the Wellcome Trust. However the manuscript has not been influenced by this association.

\section{References}

Anstey AV, Hift RJ (2007) Liver disease in erythropoietic protoporphyria: insights and implications for management. Gut 56 (7):1009-1018
Appelbaum FR (2003) The current status of hematopoietic cell transplantation. Annu Rev Med 54:491-512

Avner DL, Lee RG, Berenson MM (1981) Protoporphyrin-induced cholestasis in the isolated in situ perfused rat liver. J Clin Invest 67(2):385-394

Avner DL, Larsen R, Berenson MM (1983) Inhibition of liver surface membrane $\mathrm{Na}+, \mathrm{K}+$-adenosine triphosphatase, $\mathrm{Mg}+2$ adenosine triphosphatase and 5 '-nucleotidase activities by protoporphyrin. Observations in vitro and in the perfused rat liver. Gastroenterology 85(3):700-706

Dellon ES, Szczepiorkowski ZM, Dzik WH, Graeme-Cook F, Ades A, Bloomer JR, Cosimi AB, Chung RT (2002) Treatment of recurrent allograft dysfunction with intravenous hematin after liver transplantation for erythropoietic protoporphyria. Transplantation 73(6):911-915

Do KD, Banner BF, KATZ E, Szymanski IO, Bonkovsky HL (2002) Benefits of chronic plasmapheresis and intravenous hemealbumin in erythropoietic protoporphyria after orthotopic liver transplantation. Transplantation 73(3):469-472

Dobozy A, Csato M, Siklosi C, Simon N (1983) Transfusion therapy for erythropoietic protoporphyria. Br J Dermatol 109 (5):571-576

Doss MO, Frank M (1989) Hepatobiliary implications and complications in protoporphyria, a 20-year study. Clin Biochem 22(3):223-229

Eichbaum QG, Dzik WH, Chung RT, Szczepiorkowski ZM (2005) Red blood cell exchange transfusion in two patients with advanced erythropoietic protoporphyria. Transfusion 45(2):208-213

Elder GH, Gouya L, Whatley SD, Puy H, Badminton MN, Deybach JC (2009) The molecular genetics of erythropoietic protoporphyria. Cell Mol Biol (Noisy le grand) 55(2):118-126

Fontanellas A et al. (2000) Reversion of hepatobiliary alterations By bone marrow transplantation in a murine model of erythropoietic protoporphyria. Hepatology 32(1):73-81

Freeman RB Jr (2008) Model for end-stage liver disease (MELD) for liver allocation: a 5-year score card. Hepatology 47(3):1052-1057

Herbert A, Corbin D, Williams A, Thompson D, Buckels J, Elias E (1991) Erythropoietic protoporphyria: unusual skin and neurological problems after liver transplantation. Gastroenterology 100 (6): 1753-1757

Holme SA, Anstey AV, Finlay AY, Elder GH, Badminton MN (2006) Erythropoietic protoporphyria in the U.K.: clinical features and effect on quality of life. Br J Dermatol 155(3):574-581

Leonardi MI, Ataide EC, Boin IF, Leonardi LS (2005) Role of choledochojejunostomy in liver transplantation. Transplant Proc 37(2):1126-1128

McGuire BM et al. (2005) Liver transplantation for erythropoietic protoporphyria liver disease. Liver Transpl 11(12):1590-1596

Meerman L 2000 Erythropoietic protoporphyria. An overview with emphasis on the liver. Scand J Gastroenterol Suppl, no. 232, p. 79-85

Meerman L et al. (1999) Biliary fibrosis associated with altered bile composition in a mouse model of erythropoietic protoporphyria. Gastroenterology 117(3):696-705

Morton KO, Schneider F, Weimer MK, Straka JG, Bloomer JR (1988) Hepatic and bile porphyrins in patients with protoporphyria and liver failure. Gastroenterology 94(6):1488-1492

Neuberger J, Gimson A, Davies M, Akyol M, O'Grady J, Burroughs A, Hudson M (2008) Selection of patients for liver transplantation and allocation of donated livers in the UK. Gut 57(2):252-257

Rand EB, Bunin N, Cochran W, Ruchelli E, Olthoff KM, Bloomer JR (2006) Sequential liver and bone marrow transplantation for treatment of erythropoietic protoporphyria. Pediatrics 118(6): e1896-e1899

Reichheld JH, KATZ E, Banner BF, Szymanski IO, Saltzman JR, Bonkovsky HL (1999) The value of intravenous heme-albumin and plasmapheresis in reducing postoperative complications of 
orthotopic liver transplantation for erythropoietic protoporphyria. Transplantation 67(6):922-928

Samuel D, Boboc B, Bernuau J, BISMUTH H, Benhamou JP (1988) Liver transplantation for protoporphyria. Evidence for the predominant role of the erythropoietic tissue in protoporphyrin overproduction. Gastroenterology 95(3):816-819

Seth AK, Badminton MN, Mirza D, Russell S, Elias E (2007) Liver transplantation for porphyria: who, when, and how? Liver Transpl 13(9):1219-1227

Spiva DA, Lewis CE (1984) Erythropoietic protoporphyria: therapeutic response to combined erythrocyte exchange and plasmapheresis. Photodermatology 1(5):211-220

Todd DJ (1994) Erythropoietic protoporphyria. Br J Dermatol 131 (6):751-766

van der Meulen JH, Lewsey JD, Dawwas MF, Copley LP (2007) Adult orthotopic liver transplantation in the United Kingdom and Ireland between 1994 and 2005. Transplantation 84(5):572-579

van Wijk HJ, van Hattum J, Baart de la Faille H, van den Berg JW, Edixhoven-Bosdijk A, Wilson JH (1988) Blood exchange and transfusion therapy for acute cholestasis in protoporphyria. Dig Dis Sci 33(12):1621-1625
Wahlin S, Aschan J, Bjornstedt M, Broome U, Harper P (2007) Curative bone marrow transplantation in erythropoietic protoporphyria after reversal of severe cholestasis. J Hepatol 46(1):174-179

Wahlin S, Srikanthan N, Hamre B, Harper P, Brun A (2008) Protection from phototoxic injury during surgery and endoscopy in erythropoietic protoporphyria. Liver Transpl 14 (9): $1340-1346$

Welling TH, Heidt DG, Englesbe MJ, Magee JC, Sung RS, Campbell DA, Punch JD, Pelletier SJ (2008) Biliary complications following liver transplantation in the model for end-stage liver disease era: effect of donor, recipient, and technical factors. Liver Transpl 14(1):73-80

Whatley SD, Mason NG, Khan M, Zamiri M, Badminton MN, Missauoi WN, Dailey TA, Dailey HA, Stewart Douglas W, Wainwright NJ, Elder GH (2004) Autosomal recessive erythropoietic protoporphyria in the United Kingdom: prevalence and relationship to liver disease. J Med Genet 41(8):e105

Whatley SD et al. (2008) C-terminal deletions in the ALAS2 gene lead to gain of function and cause X-linked dominant protoporphyria without anemia or iron overload. Am J Hum Genet 83 (3):408-414 